\title{
Reading proficiency predicts the extent of the right, but not left, perceptual span in older readers
}

\author{
Aaron Veldre $^{1}$ (D) $\cdot$ Roslyn Wong ${ }^{1} \cdot$ Sally Andrews ${ }^{1}$ \\ Accepted: 16 October 2020 / Published online: 5 November 2020 \\ (C) The Psychonomic Society, Inc. 2020
}

\begin{abstract}
The gaze-contingent moving-window paradigm was used to assess the size and symmetry of the perceptual span in older readers. The eye movements of 49 cognitively intact older adults (60-88 years of age) were recorded as they read sentences varying in difficulty, and the availability of letter information to the right and left of fixation was manipulated. To reconcile discrepancies in previous estimates of the perceptual span in older readers, individual differences in written language proficiency were assessed with tests of vocabulary, reading comprehension, reading speed, spelling ability, and print exposure. The results revealed that higher proficiency older adults extracted information up to 15 letter spaces to the right of fixation, while lower proficiency readers showed no additional benefit beyond 9 letters to the right. However, all readers showed improvements to reading with the availability of up to 9 letters to the left—confirming previous evidence of reduced perceptual span asymmetry in older readers. The findings raise questions about whether the source of age-related changes in parafoveal processing lies in the adoption of a risky reading strategy involving an increased propensity to both guess upcoming words and make corrective regressions.
\end{abstract}

Keywords Reading $\cdot$ Eye movements $\cdot$ Individual differences $\cdot$ Parafoveal processing $\cdot$ Aging

Fluent online reading requires the precise coordination of perceptual, oculomotor, and language systems (Rayner, 2009). The demands of these multiple, simultaneous processes make reading susceptible to cognitive declines associated with normal aging. Older adults have deficits in visual acuityparticularly outside foveal vision (e.g., Ball, Beard, Roenker, Miller, \& Griggs, 1988) - as well as general impairments in processing speed and memory that have the potential to compromise reading (see Verhaegen, 2015, for review).

A large body of eye-movement research has established that, compared with young-adult readers, older readers tend to make more fixations of longer duration and more regressions, leading to slower overall reading time, but also to skip more words and make longer forward saccades (see Paterson et al., 2020, for review). This pattern has been attributed to a

Supplementary Information The online version contains supplementary material available at https://doi.org/10.3758/s13414-02002185-x.

Aaron Veldre

aaron.veldre@sydney.edu.au

1 School of Psychology, The University of Sydney, Sydney, NSW 2006, Australia risky reading strategy that older readers adopt to compensate for declines in lexical processing efficiency and visual acuity (Rayner, Reichle, Stroud, Williams, \& Pollatsek, 2006). The strategy is described as 'risky' because it is assumed to reflect increased reliance on top-down information from the sentence context to 'guess' upcoming words, accounting for both increases in word skipping and for corrective regressions when guesses prove to be incorrect. The risky reading hypothesis derives from evidence suggesting that older readers engage in less parafoveal processing than young readers - that is, they extract less information from outside central vision and engage in shallower preprocessing of upcoming words.

Rayner, Castelhano, and Yang (2009) used the movingwindow paradigm (McConkie \& Rayner, 1975) to assess the perceptual span of older readers. It is well-established that young adults use information from a region of approximately 3-4 letter spaces to the left and 14-15 letter spaces to the right of fixation to sustain fluent reading (see Rayner, 2014, for review). In contrast, Rayner et al. (2009) found that older adults showed no additional benefit from having two words versus one word visible to the right in a gaze-contingent fashion, but benefited from the availability of additional information to the left more than younger readers. This was interpreted as indicating that older readers have a smaller and less 
asymmetric perceptual span than younger readers. Further evidence of reduced parafoveal processing in older readers comes from studies using the boundary paradigm (Rayner, 1975), in which a target word is masked by a preview stimulus until the reader makes a saccade to it (see Schotter et al., 2012, for review). Studies comparing conditions in which the preview was valid (i.e., identical to the target) versus invalid (e.g., a random-letter string or unrelated word) have found that older readers show smaller preview effects than younger readers (Payne \& Stine-Morrow, 2012; Rayner, Castelhano, \& Yang, 2010; Rayner, Schuett, \& Slattery, 2014).

However, other research has found no difference in the size of the perceptual span between older and younger readers, raising questions about the generality of these findings. Whitford and Titone (2016) found that both older and younger bilingual readers benefited from a rightward moving window up to 14 letter spaces. Using the boundary paradigm, Risse and Kliegl (2011) found that the magnitude of the preview effect for the word two to the right of fixation was equivalent for older and younger readers.

These discrepancies in the evidence about age effects on parafoveal processing might reflect the fact that both the size of the perceptual span and the magnitude of the preview effect depend on individual differences in reading proficiency. Among skilled young-adult readers, the combination of higher reading and spelling ability is associated with a larger perceptual span and deeper parafoveal processing (Veldre \& Andrews, 2014, 2015a, 2015b, 2016; see Andrews \& Veldre, 2019, for review). If older adults' eye movements are also modulated by reading skill, it is possible that adoption of the risky reading strategy depends on proficiency: highly proficient older readers may successfully sustain efficient parafoveal processing and therefore be less likely to engage risky reading strategies. Inconsistencies in the evidence about age differences in parafoveal processing may then be due to variability in the average proficiency level of samples of older adults. ${ }^{1}$ The present research therefore investigated whether parafoveal processing in older adults is sensitive to interindividual differences in written language proficiency.

The design of the study was based on Veldre and Andrews's (2014) Experiment 2, which used the movingwindow paradigm to test the size of both the left and right perceptual span in young-adult readers for sentences varying in difficulty. The key results of the original experiment were that higher proficiency readers, as assessed by measures of reading comprehension, vocabulary, and spelling ability, showed a larger rightward perceptual span on reading speed and forward saccade length than poorer readers. However, proficiency had no impact on the use of information to the

\footnotetext{
${ }^{1}$ Inconsistent findings may also be related to differences in the visual profile of older adults because assessment of participants' visual acuity is rare (McGowan, Paterson, \& Jordan, 2013).
}

left; improvements in reading speed plateaued between 3 and 6 letter spaces to the left of fixation, consistent with previous estimates of the perceptual span (Rayner, 2014).

Testing older readers' perceptual spans using this experimental design served two primary goals. First, it compared a range of window sizes to obtain a more precise measure of the spatial extent and asymmetry of older readers' perceptual span. The evidence for reduced asymmetry in older readers appears to rest virtually entirely on Rayner et al.'s (2009) investigation of the impact of presenting a single word to the left of fixation under conditions in which all words to the right of fixation were masked. Older readers' greater sensitivity to an additional leftward word in these conditions may be specific to these unusual display conditions. To date, no studies of English readers have attempted to replicate the evidence for a larger leftward span in older readers, and a recent investigation of the perceptual span of Chinese readers found no effects of age (Xie et al., 2020). By systematically comparing three different rightward and leftward window sizes, the present study provides novel evidence about the size and symmetry of the perceptual span in older readers of English.

The second goal of the present study was to determine whether older adults' perceptual span depends on individual differences in written language proficiency by administering offline assessments of vocabulary, reading rate, reading comprehension, spelling ability, and print exposure. If older readers' reduced rightward perceptual span is primarily due to physical declines in extrafoveal visual acuity, perceptual span size should not depend on proficiency, and should be relatively independent of the difficulty of the text. However, Rayner et al. (2009) attributed older readers' reduced rightward span and enhanced leftward span to a tendency to guess upcoming words and make regressions following incorrect guesses. Highly proficient older readers might have less need to engage this strategy because they can effectively extract and use information further to the right of fixation. They may therefore show an equivalently asymmetric perceptual span to younger readers.

\section{Method}

\section{Participants}

A community sample of 49 older adults $(M=70.6$ years; range: $60-88$ years; 33 females) participated in the study for cash reimbursement. All were native English speakers with corrected-to-normal vision, which was assessed by a modified Snellen test at the experimental viewing distance. 


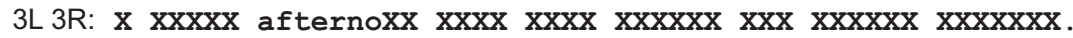 (c)

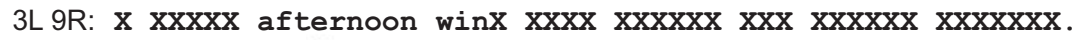 (.) \\ 3L 15R: $\mathrm{x}$ xxxxx afternoon wind blew $\operatorname{xxxxxx} \operatorname{xxx} \operatorname{xxxxxx} \operatorname{xxxxxxx}$. (c) \\ 6L 15R: $x$ xxxsh afternoon wind blew $\operatorname{xxxxxx} x x x \quad x x x x x x \quad x x x x x x x$. (c) \\ 9L 15R: $x$ fresh afternoon wind blew $\operatorname{xxxxxx} \mathrm{xxx} \operatorname{xxxxxx} \operatorname{xxxxxxx}$. (c)}

Fig. 1 Example of the critical stimuli used in the experiment with 3-letter, 9-letter, or 15-letter right windows, and 3-letter, 6-letter, or 9-letter left windows. The point of fixation is the 'e' in 'afternoon'

\section{Materials and design}

The critical stimuli were 63 pairs of sentences, averaging 10 words in length, taken from Veldre and Andrews (2014). The easy member of each pair contained a sequence of three highfrequency words (e.g., A fresh afternoon wind blew across the choppy harbour) while the hard sentence contained a lengthmatched sequence of low-frequency words (e.g., A brisk twilight gust blew across the choppy harbour; see Veldre \& Andrews, 2014, for stimulus and validation details).

The sentences were presented with a gaze-contingent moving window that displayed 3, 9, or 15 letter spaces to the right of fixation and 3, 6, or 9 letter spaces to the left of fixation. Letters outside the moving window were replaced by uppercase $X_{\mathrm{s}}$, preserving word spaces and punctuation (see Fig. 1). Nine counterbalanced lists were constructed using a Latin square design so that, across participants, all sentences appeared in all window conditions.

\section{Written language proficiency}

The written language proficiency battery included the vocabulary, reading comprehension, and reading rate subtests of the Nelson-Denny Reading Test (Brown, Fishco, \& Hanna, 1993) ${ }^{2}$; Andrews, Veldre, and Clarke's (2020) dictation and recognition tests of spelling ability; and a 50-item Author Recognition Test (Moore \& Gordon, 2015) to assess print exposure. Scores on these instruments were all moderately to highly correlated (see Table 1). The first factor from a principal component analysis, accounting for $58.77 \%$ of the variance in test scores, was therefore used as an index of overall proficiency in the analysis of the eye-movement data.

\footnotetext{
${ }^{2}$ The Vocabulary and Reading Comprehension subsections of the NelsonDenny Reading Test were administered for half the standard time limit because this procedure has been shown to yield a more normal distribution of scores (Andrews et al., 2020). Comparing the older adults' scores to the large youngadult sample $(n=345)$ reported by Andrews et al. (2020) shows that older adults were on average approximately 1.1 standard deviations higher on vocabulary, 0.9 standard deviations higher on spelling dictation, 0.8 standard deviations higher on spelling recognition, and 2.6 standard deviations higher on print exposure, but showed slightly poorer performance than young adults on reading comprehension ( $0.3 S D$ lower) and reading rate ( $0.2 S D$ lower).
}

\section{Apparatus}

Participants' eye movements were recorded by an EyeLink 1000 Plus tracker as they read sentences on a 21-in. CRT monitor (refresh rate $140 \mathrm{~Hz}$ ). The sentences were presented in black monospaced font on a gray background on a single line. Viewing was binocular, but fixation position was monitored from the right eye. A chin and forehead rest minimized head movements at a distance of $60 \mathrm{~cm}$ from the monitor, such that 2.7 characters subtended 1 degree of visual angle.

\section{Procedure}

Participants read all 126 sentences once across two counterbalanced blocks. The easy and hard version of each sentence appeared in separate blocks, each containing equal numbers of hard and easy sentences. A 3-point calibration procedure initiated each block, and three practice trials with comprehension questions preceded the randomly ordered experimental sentences. On each trial, a fixation point occupied the location of the first letter of the sentence, and the sentence was displayed when the participant made a stable fixation on this point or a new calibration procedure was performed if calibration error was greater than 0.3 degrees of visual angle. On approximately $30 \%$ of trials, the sentence was followed by a multiple-choice question that required moderate comprehension of the sentence's meaning.

\section{Results}

Mean comprehension accuracy was very high (96\%; range: $81 \%-100 \%)$. Fixations below $80 \mathrm{~ms}$ were merged with fixations within one letter space, and remaining fixations below $80 \mathrm{~ms}$ or above $1,000 \mathrm{~ms}$ were deleted ( $6.55 \%$ of fixations). Trials that were terminated early or timed out were excluded from analysis ( $<1 \%$ of trials), leaving 6,126 trials available for analysis.

Five measures of global sentence reading were analyzed (see Table 2): reading rate, measured by words per minute (WPM); forward saccade length in letter spaces; number of fixations; average fixation duration; and number of regressions. 
Table 1 Mean, standard deviation $(S D)$, range, and correlations among measures of written language proficiency

\begin{tabular}{|c|c|c|c|c|c|c|c|c|c|}
\hline Measure & Mean $(S D)$ & Min & Max & 1. & 2. & 3. & 4. & 5. & 6. \\
\hline 1. ND Vocabulary $(\max 80)$ & $68.55(10.59)$ & 37.00 & 79.00 & & & & & & \\
\hline 2. ND Comprehension $(\max 76)$ & $28.90(12.50)$ & 8.00 & 62.00 & $.62^{*}$ & & & & & \\
\hline 3. ND Reading Rate (wpm) & $295.47(83.91)$ & 139.00 & 532.00 & $.57^{*}$ & $.49^{*}$ & & & & \\
\hline 4. Spelling dictation $(\max 20)$ & $15.92(3.78)$ & 5.00 & 20.00 & $.60^{*}$ & $.43^{*}$ & $.44^{*}$ & & & \\
\hline 5. Spelling recognition $(\max 88)$ & $81.02(4.47)$ & 68.00 & 88.00 & $.56^{*}$ & $.39^{*}$ & $.40^{*}$ & $.70^{*}$ & & \\
\hline 6. Author recognition test $(\max 50)$ & $29.37(9.71)$ & 8.00 & 46.00 & $.51^{*}$ & $.50^{*}$ & $.41^{*}$ & $.47^{*}$ & $.46^{*}$ & \\
\hline 7. Overall reading proficiency $(\mathrm{PC} 1)$ & $0.00(1.88)$ & -4.18 & 3.54 & $.85^{*}$ & $.74^{*}$ & $.71^{*}$ & $.80^{*}$ & $.77^{*}$ & $.72^{*}$ \\
\hline
\end{tabular}

Note. $\mathrm{ND}=$ Nelson-Denny Reading Test (half timed); $* p<.01$ (two-tailed)

The results were analyzed using the lme4 package (Version 1.1-21; Bates, Maechler, Bolker, \& Walker, 2015) in $R$ (Version 3.6.3; R Core Team, 2020) to test linear mixedeffects models that included fixed effects of sentence difficulty, right window size, left window size, and reading proficiency. Sentence difficulty was effect coded and window size was coded as successive differences contrasts (right: 3 vs. 9 letters and 9 vs. 15 letters; left: 3 vs. 6 letters and 6 vs. 9 letters). Overall reading proficiency was included as a continuous predictor. The two-way interactions between difficulty and window size and proficiency and window size were also tested (see Table 3 for model summaries and random-effects structures).

\section{Sentence difficulty}

Participants spent longer reading hard versus easy sentences, making more fixations, longer average fixation durations and more regressions $(t \mathrm{~s}>7.01)$. However, sentence difficulty did not affect forward saccade length $(t<1)$.

\section{Written language proficiency}

High reading proficiency was associated with faster reading rate $(t=2.66)$ due to a reduced number of both fixations $(t=$ $-2.89)$ and regressions $(t=-2.15)$, but reading proficiency did not significantly affect forward saccade length or average fixation duration $(t \mathrm{~s}<1.46)$.

\section{Right window size}

Reading rate significantly increased between both the 3-letter and 9-letter windows $(t=45.31)$ and the 9-letter and 15-letter windows $(t=10.53)$. The benefit of larger rightward windows was also observed in longer forward saccades, reduced number of fixations, shorter fixation durations (all $t \mathrm{~s}>3.78$ ). There were significantly fewer regressions for the 9-letter window than the 3-letter window $(t=-17.42)$, but no further reduction in regressions at the 15-letter right window size $(t<1)$.
Sentence difficulty modulated the difference between the 3 -letter and 9-letter right window sizes on reading rate $(t=$ -3.39). As shown in Fig. 2, the impact of sentence difficulty on reading increased between the 3-letter and 9-letter windows. Sentence difficulty did not interact with right window size on any other measure (all $t \mathrm{~s}<1.86$ ).

Reading proficiency interacted with both the 3-letter versus 9-letter and 9-letter versus 15-letter window contrasts on both reading rate $(t \mathrm{~s}>2.56)$ and average fixation duration $(t \mathrm{~s}>$ 2.72 ), and with the 3-letter versus 9-letter window on fixation count $(t=2.12)$. As Fig. 3 shows, higher proficiency readers' reading speed increased up to the 15 -letter window while lower proficiency readers showed minimal increase in reading speed beyond the 9-letter window.

\section{Left window size}

Reading rate, average fixation duration, number of fixations, and number of regressions all revealed a significant benefit from having 6 compared with 3 letters visible to the left (all $t \mathrm{~s}>7.07$ ), and a further benefit from a 9-letter leftward window over a 6-letter window (all $t \mathrm{~s}>2.33$ ). However, forward saccade length was not affected by the availability of letter information to the left $(t \mathrm{~s}<1)$. Neither sentence difficulty nor reading proficiency interacted with the left window contrasts on any measure (all $t \mathrm{~s}<1.50){ }^{3}$

\footnotetext{
${ }_{3}^{3}$ Bayes factors were computed to confirm the null interactions between reading proficiency and left window size using the $1 \mathrm{mBF}$ function in the BayesFactor package (Morey et al., 2015) in R with the scaling factor for gpriors set to 0.5 and 100,000 Monte Carlo iterations. The ratio of Bayes factors for models including the two-way PC1 $\times$ Left Window Size interaction over models with additive effects of the two factors was used to evaluate evidence for or against the interaction. Bayes factors greater than 1 provide support for the interactive model, and Bayes factors below 1 provide support for the additive model. Across measures, the analyses consistently provided evidence in favor of the models containing additive effects of proficiency and left window size over the models containing the PC1 $\times$ Left Window Size interaction (all $B F \mathrm{~s}<0.29$ ). In contrast, Bayesian analyses favored models containing PC1 $\times$ Right Window Size interactions for reading rate and average fixation duration $(B F \mathrm{~S}>2.22)$ and favored models containing additive effects of the two factors for the other reading measures $(B F \mathrm{~s}<0.13)$.
} 


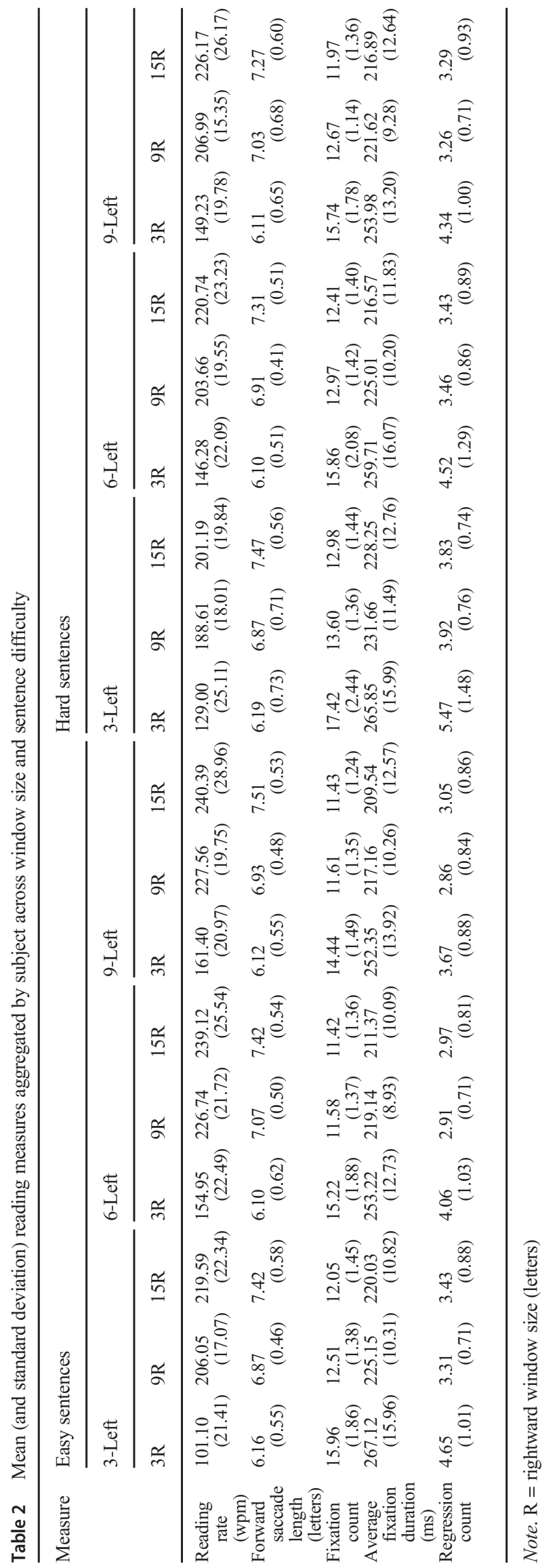

\section{Combined analyses}

To provide a direct test of age-related differences in the asymmetry of the perceptual span, we conducted a set of analyses combining the present data with the young-adult data from Veldre and Andrews's (2014) Experiment 2. The outcomes of these analyses are reported in the Supplementary Materials. The overall pattern of results showed that, on average, older adults read the sentences more slowly, skipped more words, and made more regressions than young adults. These age differences are broadly consistent with previous findings that have been interpreted as reflecting a risky reading strategy (Paterson et al., 2020). With respect to the impact of window size, older readers benefited less from the availability of information to the right of fixation than younger readers. Older readers also showed greater use of information to the left of fixation than younger readers. Thus, the combined analyses provided evidence consistent with the reduced asymmetry of perceptual span reported by Rayner et al. (2009).

\section{Discussion}

The present study used the moving-window paradigm to examine the perceptual span of older adults. Contradictory previous evidence concerning the size and symmetry of the perceptual span in older readers may reflect the impact of interindividual differences in written language proficiency that is obscured in averaged data.

The results confirmed that older adults' global reading behavior is modulated by proficiency: higher proficiency older readers had faster reading rates, made fewer fixations, and made fewer regressions. This evidence of an impact of reading skill in the restricted cohort of cognitively intact older adults who volunteer to participate in reading studies is notable, given that these individuals presumably regularly engage in reading and are likely to represent a relatively elite subset of the population. However, there was no evidence that proficiency modulated the length of forward saccades or word skipping (see Supplementary Materials) during reading - key indices of oculomotor behavior that characterize the risky reading strategy. Thus, the global reading data provided limited evidence that written language proficiency influences the specific changes in eye movements that have been identified with agerelated reading strategies.

The average data showed that older readers benefited from a rightward window up to 15 letter spaces, consistent with the findings for young readers (Rayner, 2014). The size of the right span was also found to flexibly adapt to the difficulty of the text because both the increase in reading rate and decrease in fixation duration with 9 versus 3 letters visible to the right was larger in easy than in hard sentences. Importantly, the size of the right span was modulated by proficiency 
Table 3 Results of the linear mixed-effects models

\begin{tabular}{|c|c|c|c|c|}
\hline Measure & Fixed effect & $b$ & $S E$ & $t / z$ \\
\hline \multirow[t]{15}{*}{ Reading rate } & Intercept & 193.91 & 6.31 & 30.71 \\
\hline & Sentence difficulty & -16.20 & 1.13 & -14.36 \\
\hline & Reading proficiency (PC1) & 8.30 & 3.12 & 2.66 \\
\hline & Right window size (3 vs. 9) & 62.75 & 1.38 & 45.31 \\
\hline & Right window size (9 vs. 15 ) & 14.58 & 1.38 & 10.53 \\
\hline & Left window size (3 vs. 6 ) & 17.74 & 1.38 & 12.81 \\
\hline & Left window size (6 vs. 9) & 3.31 & 1.38 & 2.39 \\
\hline & Difficulty $\times$ Right: 3 vs. 9 & -9.36 & 2.76 & -3.39 \\
\hline & Difficulty $\times$ Right: 9 vs. 15 & 3.39 & 3.15 & 1.08 \\
\hline & Difficulty $\times$ Left: 3 vs. 6 & -0.64 & 2.76 & -0.23 \\
\hline & Difficulty $\times$ Right: 6 vs. 9 & 1.07 & 2.95 & 0.36 \\
\hline & PC1 × Right: 3 vs. 9 & 3.45 & 0.76 & 4.56 \\
\hline & PC1 $\times$ Right: 9 vs. 15 & 2.21 & 0.87 & 2.56 \\
\hline & PC $1 \times$ Left: 3 vs. 6 & 1.17 & 0.78 & 1.50 \\
\hline & PC1 $\times$ Right: 6 vs. 9 & 0.04 & 0.79 & 0.06 \\
\hline \multirow[t]{15}{*}{ Forward saccade length } & Intercept & 6.83 & 0.17 & 41.09 \\
\hline & Sentence difficulty & -0.03 & 0.04 & -0.84 \\
\hline & Reading proficiency (PC1) & 0.12 & 0.09 & 1.39 \\
\hline & Right window size (3 vs. 9) & 0.82 & 0.07 & 11.31 \\
\hline & Right window size (9 vs. 15 ) & 0.45 & 0.06 & 7.24 \\
\hline & Left window size (3 vs. 6) & -0.01 & 0.04 & -0.34 \\
\hline & Left window size (6 vs. 9) & 0.01 & 0.04 & 0.27 \\
\hline & Difficulty $\times$ Right: 3 vs. 9 & -0.03 & 0.08 & -0.38 \\
\hline & Difficulty $\times$ Right: 9 vs. 15 & -0.08 & 0.08 & -0.96 \\
\hline & Difficulty $\times$ Left: 3 vs. 6 & -0.12 & 0.09 & -1.32 \\
\hline & Difficulty $\times$ Right: 6 vs. 9 & 0.04 & 0.08 & 0.49 \\
\hline & PC1 $\times$ Right: 3 vs. 9 & -0.06 & 0.04 & -1.63 \\
\hline & PC1 $\times$ Right: 9 vs. 15 & 0.01 & 0.03 & 0.22 \\
\hline & PC $1 \times$ Left: 3 vs. 6 & 0.00 & 0.02 & -0.16 \\
\hline & PC1 $\times$ Right: 6 vs. 9 & 0.01 & 0.02 & 0.66 \\
\hline \multirow[t]{15}{*}{ Fixation count } & Intercept & 13.42 & 0.47 & 28.37 \\
\hline & Sentence difficulty & 1.03 & 0.09 & 11.24 \\
\hline & Reading proficiency (PC1) & -0.72 & 0.25 & -2.89 \\
\hline & Right window size (3 vs. 9) & -3.26 & 0.11 & -28.83 \\
\hline & Right window size (9 vs. 15 ) & -0.43 & 0.11 & -3.78 \\
\hline & Left window size (3 vs. 6 ) & -0.80 & 0.11 & -7.07 \\
\hline & Left window size (6 vs. 9) & -0.28 & 0.11 & -2.46 \\
\hline & Difficulty $\times$ Right: 3 vs. 9 & 0.05 & 0.25 & 0.22 \\
\hline & Difficulty $\times$ Right: 9 vs. 15 & -0.36 & 0.27 & -1.36 \\
\hline & Difficulty $\times$ Left: 3 vs. 6 & -0.16 & 0.23 & -0.71 \\
\hline & Difficulty $\times$ Right: 6 vs. 9 & -0.04 & 0.23 & -0.18 \\
\hline & PC1 $\times$ Right: 3 vs. 9 & 0.17 & 0.08 & 2.12 \\
\hline & PC1 × Right: 9 vs. 15 & -0.03 & 0.06 & -0.53 \\
\hline & PC $1 \times$ Left: 3 vs. 6 & 0.01 & 0.08 & 0.08 \\
\hline & PC1 $\times$ Right: 6 vs. 9 & 0.05 & 0.06 & 0.74 \\
\hline \multirow[t]{4}{*}{ Average fixation duration } & Intercept & 233.03 & 3.83 & 60.92 \\
\hline & Sentence difficulty & 4.94 & 0.71 & 7.01 \\
\hline & Reading proficiency $(\mathrm{PC} 1)$ & -3.00 & 2.05 & -1.46 \\
\hline & Right window size (3 vs. 9) & -35.33 & 0.87 & -40.84 \\
\hline
\end{tabular}


Table 3 (continued)

\begin{tabular}{|c|c|c|c|c|}
\hline Measure & Fixed effect & $b$ & $S E$ & $t / z$ \\
\hline & Right window size (9 vs. 15 ) & -6.11 & 0.86 & -7.07 \\
\hline & Left window size (3 vs. 6) & -8.74 & 0.87 & -10.09 \\
\hline & Left window size (6 vs. 9) & -2.31 & 0.87 & -2.67 \\
\hline & Difficulty $\times$ Right: 3 vs. 9 & 3.33 & 1.79 & 1.86 \\
\hline & Difficulty $\times$ Right: 9 vs. 15 & 1.30 & 1.78 & 0.73 \\
\hline & Difficulty $\times$ Left: 3 vs. 6 & 1.34 & 1.73 & 0.77 \\
\hline & Difficulty $\times$ Right: 6 vs. 9 & -1.34 & 1.73 & -0.77 \\
\hline & PC1 $\times$ Right: 3 vs. 9 & -1.38 & 0.47 & -2.93 \\
\hline & PC1 × Right: 9 vs. 15 & -1.34 & 0.49 & -2.72 \\
\hline & PC1 $\times$ Left: 3 vs. 6 & -0.26 & 0.50 & -0.53 \\
\hline & PC1 $\times$ Right: 6 vs. 9 & -0.62 & 0.48 & -1.30 \\
\hline \multirow[t]{15}{*}{ Regression count } & Intercept & 3.69 & 0.28 & 13.01 \\
\hline & Sentence difficulty & 0.51 & 0.05 & 9.38 \\
\hline & Reading proficiency (PC1) & -0.33 & 0.15 & -2.15 \\
\hline & Right window size (3 vs. 9) & -1.16 & 0.07 & -17.42 \\
\hline & Right window size ( 9 vs. 15 ) & 0.05 & 0.07 & 0.76 \\
\hline & Left window size (3 vs. 6 ) & -0.53 & 0.07 & -7.97 \\
\hline & Left window size (6 vs. 9) & -0.15 & 0.07 & -2.33 \\
\hline & Difficulty $\times$ Right: 3 vs. 9 & -0.12 & 0.14 & -0.82 \\
\hline & Difficulty $\times$ Right: 9 vs. 15 & -0.16 & 0.15 & -1.08 \\
\hline & Difficulty $\times$ Left: 3 vs. 6 & -0.11 & 0.14 & -0.81 \\
\hline & Difficulty $\times$ Right: 6 vs. 9 & -0.06 & 0.13 & -0.42 \\
\hline & PC1 × Right: 3 vs. 9 & 0.01 & 0.04 & 0.30 \\
\hline & PC1 $\times$ Right: 9 vs. 15 & -0.06 & 0.04 & -1.63 \\
\hline & PC1 $\times$ Left: 3 vs. 6 & 0.00 & 0.04 & 0.07 \\
\hline & PC1 × Right: 6 vs. 9 & 0.03 & 0.04 & 0.89 \\
\hline
\end{tabular}

Note. Models with maximal random-effects structures failed to converge. The random effects were simplified by removing the correlation parameters and including by-participant and by-item random intercepts and random slopes for the highest-order within unit interaction terms (Barr, 2013; Barr, Levy, Scheepers, \& Tily, 2013) — that is, $(1+$ difficulty:right + difficulty:left $\|$ subject $)+(1+$ difficulty:right + difficulty:left + PC1:right + PC1:left $\|$ item). Significant effects are indicated in boldface

because only higher proficiency readers showed improvement on the key measure of reading rate beyond 9 letter spaces to

- Easy sentence $\mathbf{\Delta}$ Hard sentence

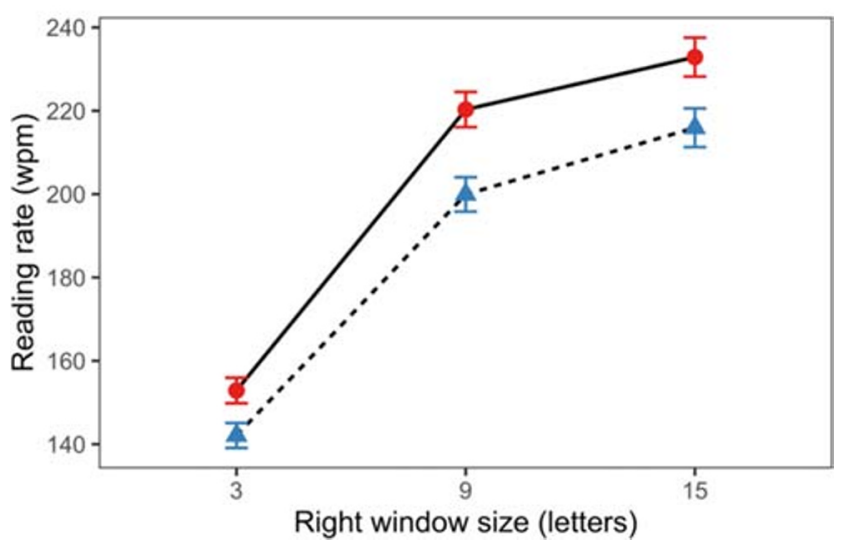

Fig. 2 Reading rate at each right window size, separately for easy and hard sentences. Error Bars are $\pm S E$ the right. This finding replicates the impact of reading proficiency on the rightward extent of the perceptual span observed among young-adult readers (Veldre \& Andrews, 2014) and demonstrates that higher proficiency older readers extract and use rightward parafoveal information as effectively as young adults.

These right-span findings contribute to explaining the discrepancy in previous estimates of the perceptual span in older readers. Whitford and Titone (2016) speculated that their failure to observe the age-related reductions in perceptual span reported by Rayner et al. (2009) may reflect differences in the average proficiency level of the older samples in the two studies, which were not directly assessed. The present findings confirm that proficiency affects the extent of older adults' rightward span, indicating that differences in average proficiency between older samples could account for discrepant conclusions about the effect of age on parafoveal processing. 
- Low proficiency $\boldsymbol{\Delta}$ High proficiency

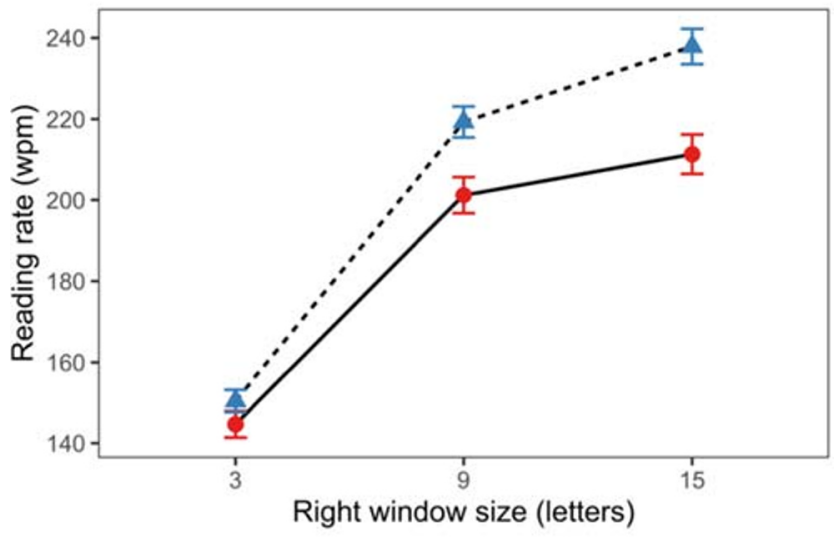

- Low proficiency $\boldsymbol{\Delta}$ High proficiency

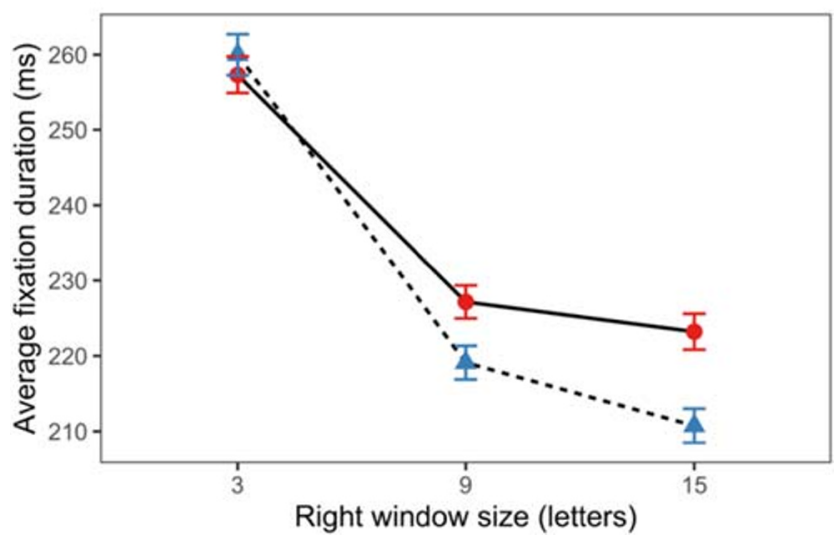

Fig. 3 Reading rate (upper panel) and average fixation duration (lower panel) at each right window size, separately for participants below and above the median on overall reading proficiency. Error bars are $\pm S E$

In contrast to the right-span findings, the impact of varying the availability of information to the left on reading suggested that all older adults benefit from an enlarged leftward window, regardless of proficiency. There were improvements to all reading measures, except forward saccade length, when the left window size increased from 3 to 6 letters and further improvements on these measures with an increase from 6 to 9 letters to the left. The latter effect contrasts with Veldre and Andrews's (2014) finding that young readers showed no improvement in reading speed from the availability of more than 6 letters to the left of fixation, consistent with previous conclusions that young readers typically do not use information to the left beyond the fixated word (Rayner, 2014).

The present data converge with Rayner et al.'s (2009) conclusion that older adults use more information to the left of fixation than younger adults and demonstrate that this effect generalizes from their limited display conditions. The present finding that older readers showed an additional benefit from a 9-letter relative to a 6-letter leftward window across a range of rightward window sizes provides stronger evidence for the reduced asymmetry of older adults' perceptual span. The combined analyses also converged with Rayner et al.'s (2009) data in showing that, on average, older readers have a less asymmetric perceptual span than younger readers. Notably, regression rate decreased rather than increased with leftward window size, suggesting that the availability of information to the left of fixation enhanced reading fluency without increasing the likelihood of fixating those regions. However, unlike rightward window size, leftward spatial extent did not interact with either sentence difficulty or proficiency on any measure.

Thus, the present results demonstrate two effects of age on perceptual span that appear to be independent. Overall, older adults benefited more from a wider leftward window than the young adults tested on the same materials by Veldre and Andrews (2014) and participants in previous moving-window studies (Rayner, 2014), confirming Rayner et al.'s (2009) claim that older readers have a less asymmetric perceptual span. However, the individual-differences results of the present study demonstrate that reduced rightward perceptual span size is restricted to older readers with lower reading proficiency. Thus, the processes responsible for the age effects on leftward span appear to be different from those underlying rightward parafoveal effects because only the latter are sensitive to processing difficulty and reading proficiency. On the one hand, these findings are broadly compatible with the view that older readers use information from a more symmetrically distributed region of text around fixation during reading than young adults. However, we did not find evidence to support the risky reading account's assumption that the same unconscious guessing strategy is responsible for both reduced extraction of information from rightward parafoveal words and increased leftward span associated with an increased rate of regressions required when risky guesses are disconfirmed. A contextually based guessing strategy should be sensitive to both stimulus difficulty and individual differences in proficiency, and these factors did yield the expected effects on rightward span. The absence of parallel effects on leftward span suggests that older readers' increased use of information from this region may reflect a general tendency to engage in late confirmatory processes to verify comprehension rather than reanalysis triggered by disconfirmed risky guesses (Christianson, Luke, Hussey, \& Wochna, 2017). Further research manipulating the type of parafoveal preview information available about upcoming words will provide more refined insight into the source of age-related changes to reading behavior.

Author note We thank Kelly Dann for assistance with data collection, and Adam Parker and two anonymous reviewers for helpful comments on a previous version of this article. This research was supported under Australian Research Council's Discovery Projects funding scheme (Grant Number DP18102705). The data and stimulus materials from this study are available at osf.io/24zhp. The study was not preregistered. 


\section{References}

Andrews, S., \& Veldre, A. (2019). What is the most plausible account of the role of parafoveal processing in reading? Language and Linguistics Compass, 13, e12344.

Andrews, S., Veldre, A., \& Clarke, I. E. (2020). Measuring lexical quality: The role of spelling ability. Behavior Research Methods. https:// doi.org/10.3758/s13428-020-01387-3

Ball, K. K., Beard, B. L., Roenker, D. L., Miller, R. L., \& Griggs, D. S. (1988). Age and visual search: Expanding the useful field of view. Journal of the Optical Society of America A, 5, 2210-2219.

Barr, D. J. (2013). Random effects structure for testing interactions in linear mixed-effects models. Frontiers in Psychology, 4, 328.

Barr, D. J., Levy, R., Scheepers, C., \& Tily, H. J. (2013). Random effects structure for confirmatory hypothesis testing: Keep it maximal. Journal of Memory and Language, 68, 255-278.

Bates, D., Maechler, M., Bolker, B., \& Walker, S. (2015). Fitting linear mixed-effects models using lme4. Journal of Statistical Software, 67, 1-48.

Brown, J. I., Fishco, V. V., \& Hanna, G. (1993). Nelson-Denny Reading Test. Austin, TX: PRO-ED, Inc.

Christianson, K., Luke, S. G., Hussey, E. K., \& Wochna, K. L. (2017). Why reread? Evidence from garden-path and local coherence structures. Quarterly Journal of Experimental Psychology, 70, 13801405.

McConkie, G. W., \& Rayner, K. (1975). The span of the effective stimulus during a fixation in reading. Perception \& Psychophysics, 17, $578-586$.

McGowan, V. A., Paterson, K. B., \& Jordan, T. R. (2013). Age-related visual impairments and perceiving linguistic stimuli: The rarity of assessing the visual abilities of older participants in written language research. Experimental Aging Research, 39, 70-79.

Moore, M., \& Gordon, P. C. (2015). Reading ability and print exposure: item response theory analysis of the author recognition test. Behavior Research Methods, 47, 1095-1109.

Paterson, K. B., McGowan, V. A., Warrington, K. L., Li, L., Li, S., Xie, F., ... Wang, J. (2020). Effects of normative aging on eye movements during reading. Vision, 4, 7.

Payne, B. R., \& Stine-Morrow, E. A. L. (2012). Aging, parafoveal preview, and semantic integration in sentence processing: Testing the cognitive workload of wrap-up. Psychology and Aging, 27, 638649.

R Core Team. (2020). R: A language and environment for statistical computing. Vienna, Austria: R Foundation for Statistical Computing. Retrieved from https://www.R-project.org/

Rayner, K. (1975). The perceptual span and peripheral cues in reading. Cognitive Psychology, 7, 65-81.

Rayner, K. (2009). Eye movements and attention in reading, scene perception, and visual search. Ouarterly Journal of Experimental Psychology, 62, 1457-1506.
Rayner, K. (2014). The gaze-contingent moving window in reading: Development and review. Visual Cognition, 22, 242-258.

Rayner, K., Castelhano, M. S., \& Yang, J. (2009). Eye movements and the perceptual span in older and younger readers. Psychology and Aging, 24, 755-760.

Rayner, K., Castelhano, M. S., \& Yang, J. (2010). Preview benefit during eye fixations in reading for older and younger readers. Psychology and Aging, 25, 714-718.

Rayner, K., Reichle, E. D., Stroud, M. J., Williams, C. C., \& Pollatsek, A. (2006). The effect of word frequency, word predictability, and font difficulty on the eye movements of young and older readers. Psychology and Aging, 21, 448-465.

Rayner, K., Yang, J., Schuett, S., \& Slattery, T. J. (2014). The effect of foveal and parafoveal masks on the eye movements of older and younger readers. Psychology and Aging, 29, 205-212.

Risse, S., \& Kliegl, R. (2011). Adult age differences in the perceptual span during reading. Psychology and Aging, 26, 451-460.

Schotter, E. R., Angele, B., \& Rayner, K. (2012). Parafoveal processing in reading. Attention, Perception and Psychophysics, 74, 5-35.

Veldre, A., \& Andrews, S. (2014). Lexical quality and eye movements: Individual differences in the perceptual span of skilled adult readers. Quarterly Journal of Experimental Psychology, 67, 703-727.

Veldre, A. \& Andrews, S. (2015a). Parafoveal lexical activation depends on skilled reading proficiency. Journal of Experimental Psychology: Learning Memory and Cognition, 41, 586-595.

Veldre, A. \& Andrews, S. (2015b). Parafoveal lexical activation is modulated by the precision of skilled readers' lexical representations. Journal of Experimental Psychology: Human Perception and Performance, 41, 219-232.

Veldre, A., \& Andrews, S. (2016). Semantic preview benefit in English: Individual differences in the extraction and use of parafoveal semantic information. Journal of Experimental Psychology: Learning, Memory, and Cognition, 42, 837-854.

Verhaegen, P. (2015). Cognitive aging. In D. Reisberg (Ed)., The Oxford handbook of cognitive psychology. Oxford, UK: Oxford University Press.

Whitford, V., \& Titone, D. (2016). Eye movements and the perceptual span during first- and second-language sentence reading in bilingual older adults. Psychology and Aging, 31, 58-70.

Xie, F., McGowan, V. A., Chang, M., Li, L., White, S. J., Paterson, K. B., Wang, J., \& Warrington, K. L. (2020). Revealing similarities in the perceptual span of young and older Chinese readers. Quarterly Journal of Experimental Psychology, 47(8). https://doi.org/10. $1177 / 1747021819899826$

Publisher's note Springer Nature remains neutral with regard to jurisdictional claims in published maps and institutional affiliations. 